\title{
Prevalence of Sexual Dysfunctions: A Systemic Approach
}

\author{
Azita Goshtasebi ${ }^{1}$, Samira Behboudi Gandevani², \\ and Abbas Rahimi Foroushani ${ }^{3}$ \\ ${ }^{1}$ Department of Family Health, Mother and Child Health Research Center, \\ Iranian Institute for Health Sciences Research, ACECR, Tehran, \\ ${ }^{2}$ Midwifery Department, Faculty of Medicine, Tarbiat Modares University, Tehran, \\ ${ }^{3}$ Department of Epidemiology and Biostatistics, Faculty of Public Health, Tehran, \\ University of Medical Sciences, Tehran, \\ Iran
}

\section{Introduction}

This chapter is intended to provide an evidence-based overview of the worldwide epidemiology of sexual dysfunction since the 1990s in general populations of different countries, allowing for the generalization of findings at the given population level. The descriptive and analytic literature on sexual function was identified through searching conventional databases, literature surveys and references. This chapter is organized as follows: we first review epidemiological concepts focusing on the issue of determining prevalence; then, we review the female and male sexual dysfunctions prevalence.

Since only recently sexual function and sexual problems have been openly discussed in most societies and cultures (Tiefer, 2001), few epidemiologic data exist until the middle of the twentieth century. The large population-based study of normative data on female sexuality was published by Kinsey and coworkers in 1953. Recent studies, however, have presented a more accurate picture of sexual dysfunction prevalence.

\section{The epidemiology of sexual dysfunction}

Epidemiology is a scientific study of the distribution and determinants of diseases in populations. Epidemiological data are the basis for assessing the overall impact of a condition on a given society (Prins et al., 2002). These data are needed for public health systems in order to recognize the impact of the studied condition in the population and organize screening, diagnostic and treatment strategies .One of the basic epidemiological measures of outcome occurrence is prevalence which is defined as the proportion of a population exhibiting a health condition during a specific time interval (Simons et al. 2001). Moreover, prevalence is characterized by the proportion of a given population which has the condition at a given time. While prevalence can refer to any time period, researchers typically distinguish among point, period and lifetime prevalence. An important conceptual issue is to define sexual dysfunction which is used when it is clinically diagnosed. Another 
issue is to select the study sample. Community-based samples are the most appropriate ones which define the potential number of patients sustaining the disorder/condition who might benefit from treatment. The study sample must be a representative of the studied population in terms of social, cultural and health status. And, the last one is to select the tools used for screening.

In this chapter, the criterion was the prevalence estimate for general populations, which included a representative sample of community with overall methodological quality. The internal validity of the study was assessed by data collection procedures, measurement instruments, defining health conditions and informative content of reported prevalence. External validity was assessed by the generalizability of the study and source of population, sampling, eligibility of criteria and response rate (Tsai et al, 2011).

The second important issue is that sexual dysfunction may be best conceptualized as the global inhibition of sexual response due to interpersonal factors (Hartmann et al, 2002) because many cases of sexual dysfunction can be regarded as the adaption to sexual relationship problems. In other words, sexual dysfunction must be seen in a multi-faceted socio-psycho-biologic context. Therefore, there are different scales for defining and classifying sexual dysfunction including the American Psychiatric Association's Diagnosis and Statistical Manual for Mental Disorder, $4^{\text {th }}$ text revision (DSM-III or IV-TR), the World Health Organization's International Classification of Diagnosis (ICD-10), Profile of Female Sexual Function(PFSF), Female Sexual Function Index (FSFI), Golombok Rust Inventory of Sexual Satisfaction (GRISS), International Index of Erectile Function (IIEF), Sexual Function Questionnaire (SFQ) and other researcher-made validated questionnaires . Clearly, the type of applied definition affects the prevalence estimate of sexual problems. Although the database in both groups is rapidly growing, in this chapter, we tried to include all the recent studies of the field.

We focused on epidemiologic studies on sexual problems, sexual disorders or sexual distress which have estimated prevalence of one or more sexual problems for general populations in the world. Thus, we excluded studies with a small sample size and studies in special populations like specific health conditions.

In this review, we searched MEDLINE, SCIENCEDIRECT, PUBMED, GOOGLE SCHOLAR, JSTORE and JSTORE PROQUEST using the following key words: epidemiology, prevalence plus sexual dysfunction, sexual function disturbance, sexual disorder, dyspareunia, vaginismus, anorgasmia, lack of lubrication, sexual arousal, sexual desire, hypoactive sexual desire disorder, sexual aversion disorder, orgasmic disorder, erectile dysfunction, early ejaculation and premature ejaculation.

\section{Prevalence of female sexual dysfunction}

Despite increasing scientist interest in female sexual difficulty and dysfunction, the true prevalence of female sexual dysfunctions (FSD) in the general population remains a contentious issue. One reason is the great deal of variation in the published prevalence estimates of female sexual difficulties/ dysfunctions. This variation may be due, in part, to real differences among populations, and the way FSD is measured (Lindau et al,. 2007). Lack of standardization of outcome measures is an important issue in the FSD literature which has been raised by previous authors. Also, different time frames have influenced the prevalence rate. If the period of study increases, prevalence increases, too (Mercer et al,. 2003). 
The prevalence of female sexual dysfunctions, as reported in reasonably valid descriptive investigations, are showed in Tables 1-4. There are currently four international data sets with some information about women's sexual problem; five studies in Africa, eleven studies in Asia, eleven studies in Europe and eleven studies in America.

\subsection{Sexual interest/desire dysorders}

Table 1 show that the low level of sexual desire prevails in $11.2 \%-66.4 \%$ of subjects in different age strata. This indicates that sexual arousal dysfunction with this large variation is a worldwide problem at different ages. In several countries, there is a clear decline in sexual interest at advanced ages.

\subsection{Arousal/lubrication dysorders}

There are genital and psychological aspects for arousal disorders. But, they are not explicitly separated. Insufficient lubrication generally appears in almost $49 \%$ of women. Also, it seems that this problem is more common in two ends of reproductive ages.

\subsection{Orgasm dysorders}

The prevalence of orgasmic dysfunction varies considerably within and between different geographic areas and some researchers believe that this problem may or may not be agedependent. The highest report belongs to India in which more than $86 \%$ of women report this problem. Also, this problem is very common among African women.

\subsection{Dyspareunia}

The manifest of genital pain during intercourse has been also reported by a large number of women all over the world. Overall, high prevalence of about $64 \%$ has been found in Asia and Africa.

Vaginismus is another painful condition during intercourse with high prevalence in Asia. But, it appears that there is clear lack of investigation of this problem in the world.

\section{Prevalence of male sexual dysfunction}

Male sexual dysfunction includes erectile dysfunction (ED), ejaculation disorders, orgasmic dysfunctions and disorders of sexual interest/desire. Epidemiologic studies have supported the high prevalence of male sexual dysfunction worldwide; however, the data are limited. Many of the epidemiologic studies are old and related to poor methodology. In this chapter, we reviewed 29 multiethnic studies about these problems. Tables 1-4 show validated studies on the prevalence of male sexual dysfunction.

\subsection{Erectile dysfunction (ED)}

Erectile dysfunction (ED) is defined as the consistent or recurrent inability of a man to attain and/or maintain penile erection sufficiently for a sexual activity. A 3-month minimum duration is accepted for the establishment of the diagnosis. Several studies have provided data on the prevalence of ED. The prevalence of ED on a worldwide basis has a great deal of variation around $9 \%-69 \%$. And, there is a clear increase of this disorder at older ages. In all studies, ED has a rather high rate from $20 \%$ to $40 \%$ for the ages 60 to 69 years old, some increasing after the age of 65 years old. 


\subsection{Ejaculation disorders}

Ejaculation disorders include early ejaculation, delayed ejaculation and anejaculation. The term early ejaculation is used to replace premature ejaculation, a term considered relatively inaccurate and pejorative. Early ejaculation is the ejaculation that occurs sooner than desired, either before or shortly after penetration, over which the sufferer has minimal or no control. Like all or most other dysfunctions, this is primarily a self-reported diagnosis. Delayed ejaculation is the undue delay in reaching a climax during sexual activity. Anejaculation is the absence of ejaculation during orgasm (Althof et al, 2006).

The major problem in assessing the prevalence of early ejaculation is lack of an accurate (validated) definition. It can be defined by time of ejaculation, in the context of the sufferer's or partner's satisfaction, the number of penile thrusts after intromission or even in the context of the amount of sexual stimulation. Similarly, there is lack of definition for the delayed ejaculation. The highest prevalence rate of 31\% (men aged 18-59 years old) was given by the NHSLS study in the United States (Laumann et al., 1999). In the sub-groups aged 18 to 29,30 to 39,40 to 49 and 50 to 59 years old, the prevalence was $30 \%, 32 \%, 28 \%$ and $55 \%$, respectively. These high prevalence rates may be a result of the dichotomous scale (yes/no) in a single question asking whether the ejaculation occurred too early or not.

\subsection{Orgasmic dysfunction}

Orgasmic dysfunction is the inability in achieving orgasm, markedly diminished intensity of orgasmic sensations or marked delay of orgasm during conscious sexual activity. There is a self-report of high sexual arousal/excitement in this disorder. Prevalence data on orgasmic dysfunction are scarce and report 5\%-33\% of all men in the world. One simple reason explaining the difficulty of assessing the prevalence of orgasmic dysfunction is that some men may be unable to distinguish between ejaculation and orgasm.

\subsection{Sexual interest/desire dysfunctions}

Sexual interest/desire dysfunctions are diminished or no feelings of sexual interest or desire, no sexual thoughts or fantasies and lack of responsive desire. This problem has been neglected in epidemiologic studies to some extent; but it is quite commonly seen in clinical practices. The prevalence rate of sexual interest disorders is $11 \%-28 \%$ around the world. The highest prevalence rate of sexual interest disorders was reported in a study conducted in Asia in men aged 40 to 80 years old. It seems that there is not any pronounced age effect on this problem. However, more research would shed more light on this issue.

\begin{tabular}{|c|c|c|c|c|c|}
\hline & Problem or question & $\begin{array}{c}\text { Sample } \\
\text { size }\end{array}$ & Scale & $\begin{array}{c}\text { Cohort } \\
\text { age } \\
\text { (year) }\end{array}$ & $\begin{array}{c}\text { Prevalence } \\
(\%)\end{array}$ \\
\hline \multicolumn{6}{|l|}{ Female } \\
\hline Amidu et al, 2011 & $\begin{array}{c}\text { Overall sexual problems } \\
\text { Anorgasmia } \\
\text { Sexual infrequency } \\
\text { Dissatisfaction } \\
\text { Vaginismus } \\
\text { Avoidance of sexual intercourse }\end{array}$ & 301 & GRISS & $18-58$ & $\begin{array}{l}72.8 \\
72.4 \\
71.4 \\
77.7 \\
68.1 \\
62.5\end{array}$ \\
\hline
\end{tabular}




\begin{tabular}{|c|c|c|c|c|c|}
\hline Laumann et al, 2005 & $\begin{array}{l}\text { Lack of sexual interest } \\
\text { Inability to reach orgasm } \\
\text { Orgasm too quickly } \\
\text { Pain during sex } \\
\text { Lubrication difficulties }\end{array}$ & 967 & $\begin{array}{c}\text { Ad hoc } \\
\text { questionnaire }\end{array}$ & $40-80$ & $\begin{array}{c}43.4 \\
23 \\
10 \\
21 \\
23 \\
\end{array}$ \\
\hline Elnashar et al, 2007 & $\begin{array}{c}\text { Dyspareunia } \\
\text { Decreased sexual desire } \\
\text { Difficult arousal } \\
\text { Anorgasmia } \\
\end{array}$ & 936 & $\begin{array}{c}\text { Ad hoc } \\
\text { questionnaire }\end{array}$ & $16-49$ & $\begin{array}{c}31.5 \\
49.6 \\
36 \\
16.9 \\
\end{array}$ \\
\hline Kadri et al, 2002 & $\begin{array}{c}\text { Overall sexual dysfunction } \\
\text { Hypoactive sexual desire } \\
\text { disorder } \\
\text { Sexual aversion disorder } \\
\text { Orgasmic disorder } \\
\text { Sexual arousal disorder } \\
\text { Dyspareunia } \\
\text { Vaginismus }\end{array}$ & 728 & DSM-IV & $20-80$ & $\begin{array}{c}26.6 \\
18.3 \\
15 \\
12 \\
8.3 \\
7.5 \\
6.2\end{array}$ \\
\hline Hassanin et al, 2010 & $\begin{array}{l}\text { Overall sexual dysfunction } \\
\text { Low sexual desire } \\
\text { Dyspareunia } \\
\text { Sexual dissatisfaction } \\
\text { Lack of lubrication } \\
\text { Low sexual arousal } \\
\text { Orgasmic disorder }\end{array}$ & 601 & $\begin{array}{c}\text { Ad hoc } \\
\text { questionnaire }\end{array}$ & $18-60$ & $\begin{array}{c}76.9 \\
66.4 \\
64 \\
54 \\
53 \\
57 \\
61 \\
\end{array}$ \\
\hline \multicolumn{6}{|l|}{ Male } \\
\hline Seyam et al, 2003 & Erectile dysfunction & 805 & $\begin{array}{c}\text { Ad hoc } \\
\text { questionnaire }\end{array}$ & $\begin{array}{c}20+ \\
50-59 \\
60-69 \\
70-79 \\
\end{array}$ & $\begin{array}{c}10.3 \\
26 \\
49 \\
52 \\
\end{array}$ \\
\hline Amidu et al, 2011 & $\begin{array}{c}\text { Overall sexual dysfunction } \\
\text { Premature ejaculation } \\
\text { Impotency } \\
\text { No sensuality } \\
\text { Avoidance }\end{array}$ & 255 & GRISS & $19+$ & $\begin{array}{c}66 \\
64.7 \\
59.6 \\
59.2 \\
49\end{array}$ \\
\hline
\end{tabular}

GRISS: Golombok Rust Inventory of Sexual Satisfaction

DSM-IV: Diagnosis and Statistical Manual for Mental Disorder, $4^{\text {th }}$

Table 1. Characteristics of African studies of sexual dysfunction

\begin{tabular}{|c|c|c|c|c|c|}
\hline & Problem or question & $\begin{array}{c}\text { Sample } \\
\text { size }\end{array}$ & Scale & $\begin{array}{c}\text { Cohort } \\
\text { age } \\
\text { (year) }\end{array}$ & $\begin{array}{c}\text { Prevalence } \\
(\%)\end{array}$ \\
\hline \multicolumn{6}{|c|}{ Female } \\
\hline $\begin{array}{c}\text { Shifren et al, } \\
2008\end{array}$ & Low desire & 31581 & CSFQ-14 & $18+$ & 37.7 \\
\hline
\end{tabular}




\begin{tabular}{|c|c|c|c|c|c|}
\hline & $\begin{array}{c}\text { Low arousal } \\
\text { Low orgasm } \\
\text { Any (desire, arousal or orgasm) } \\
\end{array}$ & & & & $\begin{array}{l}25.3 \\
21.1 \\
43.1\end{array}$ \\
\hline $\begin{array}{c}\text { Lindau et al, } \\
2007\end{array}$ & $\begin{array}{c}\text { Low desire } \\
\text { Difficulty with vaginal lubrication } \\
\text { Inability to climax }\end{array}$ & 1550 & $\begin{array}{c}\text { Ad hoc } \\
\text { questionnaire }\end{array}$ & $57-85$ & $\begin{array}{l}43 \\
39 \\
34\end{array}$ \\
\hline $\begin{array}{c}\text { Laumann et al, } \\
2005\end{array}$ & $\begin{array}{l}\text { Lack of sexual interest } \\
\text { Inability to reach orgasm } \\
\text { Orgasm too quickly } \\
\text { Pain during sex } \\
\text { Pain during sex }\end{array}$ & 1845 & $\begin{array}{c}\text { Ad hoc } \\
\text { questionnaire }\end{array}$ & $40-80$ & $\begin{array}{c}32.9 \\
25.2 \\
10.5 \\
14 \\
27.1\end{array}$ \\
\hline $\begin{array}{c}\text { Bancroft et al, } \\
2002\end{array}$ & $\begin{array}{l}\text { Lubrication problems } \\
\text { Pain disorders } \\
\text { No orgasm } \\
\end{array}$ & 987 & DSM-IV & $20-65$ & $\begin{array}{l}31 \\
3 \\
9 \\
\end{array}$ \\
\hline Addis et al, 2006 & Overall sexual dysfunction & 2109 & $\begin{array}{c}\text { Ad hoc } \\
\text { questionnaire }\end{array}$ & $40-69$ & 24 \\
\hline \multirow[t]{3}{*}{$\begin{array}{c}\text { Laumann et al, } \\
2008\end{array}$} & Lacked interest in sex & 1550 & $\begin{array}{c}\text { Ad hoc } \\
\text { questionnaire }\end{array}$ & $\begin{array}{l}57-64 \\
65-74 \\
75-85\end{array}$ & $\begin{array}{l}45.4 \\
37.6 \\
49.3 \\
\end{array}$ \\
\hline & Unable to achieve orgasm & & & $\begin{array}{l}57-64 \\
65-74 \\
75-85\end{array}$ & $\begin{array}{c}35 \\
33.4 \\
38.2\end{array}$ \\
\hline & Experienced pain during sex & & & $\begin{array}{l}57-64 \\
65-74 \\
75-85\end{array}$ & $\begin{array}{l}18.2 \\
18.9 \\
11.8\end{array}$ \\
\hline Abdo et al, 2004 & $\begin{array}{l}\text { Lack of sexual desire } \\
\text { Pain during sexual intercourse } \\
\text { Orgasmic dysfunction }\end{array}$ & 1219 & $\begin{array}{c}\text { Ad hoc } \\
\text { questionnaire }\end{array}$ & $18+$ & $\begin{array}{c}26.7 \\
23.1 \\
21 \\
\end{array}$ \\
\hline \multirow[t]{5}{*}{$\begin{array}{c}\text { Laumann et al, } \\
1999\end{array}$} & Overall sexual dysfunction & 1749 & DSM-IV & $18-59$ & 43 \\
\hline & Lacked Interest in Sex & & & $\begin{array}{l}18-29 \\
30-39 \\
40-49 \\
50-59\end{array}$ & $\begin{array}{l}32 \\
32 \\
30 \\
27\end{array}$ \\
\hline & Unable to Achieve Orgasm & & & $\begin{array}{l}18-29 \\
30-39 \\
40-49 \\
50-59\end{array}$ & $\begin{array}{l}26 \\
28 \\
22 \\
23\end{array}$ \\
\hline & Experienced Pain During Sex & & & $\begin{array}{l}18-29 \\
30-39 \\
40-49 \\
50-59\end{array}$ & $\begin{array}{c}21 \\
15 \\
13 \\
8\end{array}$ \\
\hline & Difficulty in Lubricating & & & $\begin{array}{l}18-29 \\
30-39 \\
40-49 \\
50-59\end{array}$ & $\begin{array}{l}19 \\
18 \\
21 \\
27 \\
\end{array}$ \\
\hline
\end{tabular}




\begin{tabular}{|c|c|c|c|c|c|}
\hline West et al, 2008 & $\begin{array}{c}\text { Low Sexual Desire } \\
\text { Hypoactive Sexual Desire } \\
\text { Disorder }\end{array}$ & 2207 & PFSF & $30-70$ & $\begin{array}{c}36.2 \\
8.3\end{array}$ \\
\hline Junior et al, 2005 & $\begin{array}{l}\text { Lubrication difficulties } \\
\text { Lack of sexual interest }\end{array}$ & 728 & $\begin{array}{c}\text { Ad hoc } \\
\text { questionnaire }\end{array}$ & $40-80$ & $\begin{array}{l}23.4 \\
22.7\end{array}$ \\
\hline \multicolumn{6}{|l|}{ Male } \\
\hline $\begin{array}{c}\text { Lindau et al, } \\
2007\end{array}$ & Erectile difficulties & 1455 & $\begin{array}{c}\text { Ad hoc } \\
\text { questionnaire }\end{array}$ & $57-85$ & 37 \\
\hline \begin{tabular}{|c|} 
Laumann et al, \\
2005
\end{tabular} & $\begin{array}{l}\text { Lack of sexual interest } \\
\text { Inability to reach orgasm } \\
\text { Early ejaculation } \\
\text { Pain during sex } \\
\text { Erectile difficulties }\end{array}$ & 2205 & $\begin{array}{c}\text { Ad hoc } \\
\text { questionnaire }\end{array}$ & $40-80$ & $\begin{array}{c}17.6 \\
14.5 \\
27.4 \\
3.6 \\
20.6\end{array}$ \\
\hline \multirow[t]{3}{*}{$\begin{array}{c}\text { Laumann et al, } \\
2008\end{array}$} & Lacked interest in sex & 1550 & $\begin{array}{c}\text { Ad hoc } \\
\text { questionnaire }\end{array}$ & $\begin{array}{l}57-64 \\
65-74 \\
75-85\end{array}$ & $\begin{array}{l}27.8 \\
28.7 \\
24.4\end{array}$ \\
\hline & Unable to achieve orgasm & & & $\begin{array}{l}57-64 \\
65-74 \\
75-85\end{array}$ & $\begin{array}{c}16.1 \\
22.9 \\
33\end{array}$ \\
\hline & Experienced pain during sex & & & $\begin{array}{l}57-64 \\
65-74 \\
75-85\end{array}$ & $\begin{array}{c}3 \\
3.2 \\
1\end{array}$ \\
\hline Derby et al, 2000 & Erectile dysfunction & 505 & $\begin{array}{c}\text { IIEF } \\
\text { BMSFI }\end{array}$ & $50-70$ & $\begin{array}{c}18 \\
8\end{array}$ \\
\hline $\begin{array}{l}\text { Ansong et al, } \\
2000\end{array}$ & Erectile dysfunction & 5198 & $\begin{array}{c}\text { Ad hoc } \\
\text { questionnaire }\end{array}$ & $\begin{array}{l}50-76 \\
50-54 \\
55-59 \\
60-64 \\
65-69 \\
70-76 \\
\end{array}$ & $\begin{array}{c}46.3 \\
26 \\
34.9 \\
46.9 \\
57.8 \\
69.4 \\
\end{array}$ \\
\hline $\begin{array}{l}\text { Moreira et al, } \\
2001\end{array}$ & Erectile dysfunction & 1286 & $\begin{array}{c}\text { Ad hoc } \\
\text { questionnaire }\end{array}$ & $18+$ & 46.2 \\
\hline Saigal et al, 2006 & Erectile dysfunction & 3566 & $\begin{array}{c}\text { Ad hoc } \\
\text { questionnaire }\end{array}$ & $\begin{array}{c}20+ \\
20-29 \\
30-39 \\
40-49 \\
50-59 \\
60-69 \\
70-74 \\
\geq 75\end{array}$ & $\begin{array}{c}12.3 \\
4.7 \\
3.4 \\
7 \\
19.9 \\
27 \\
38 \\
30\end{array}$ \\
\hline $\begin{array}{c}\text { Johannes et al, } \\
2000\end{array}$ & Erectile dysfunction & 1156 & $\begin{array}{c}\text { Ad hoc } \\
\text { questionnaire }\end{array}$ & $\begin{array}{l}40-69 \\
40-49 \\
50-59 \\
60-69\end{array}$ & $\begin{array}{l}25.9 \\
12.4 \\
29.8 \\
46.4\end{array}$ \\
\hline
\end{tabular}




\begin{tabular}{|c|c|c|c|c|c|}
\hline $\begin{array}{c}\text { Laumann et al, } \\
1999\end{array}$ & Overall sexual dysfunction & 1410 & DSM-IV & $18-59$ & 31 \\
\hline & Lacked interest in sex & & & $\begin{array}{l}18-29 \\
30-39 \\
40-49 \\
50-59\end{array}$ & $\begin{array}{l}14 \\
13 \\
15 \\
17\end{array}$ \\
\hline & Unable to achieve orgasm & & & $\begin{array}{l}18-29 \\
30-39 \\
40-49 \\
50-59\end{array}$ & $\begin{array}{l}7 \\
7 \\
9 \\
9\end{array}$ \\
\hline & Climax too early & & & $\begin{array}{l}18-29 \\
30-39 \\
40-49 \\
50-59\end{array}$ & $\begin{array}{l}30 \\
32 \\
28 \\
31\end{array}$ \\
\hline & $\begin{array}{l}\text { Trouble maintaining or } \\
\text { Achieving an erection }\end{array}$ & & & $\begin{array}{l}18-29 \\
30-39 \\
40-49 \\
50-59\end{array}$ & $\begin{array}{c}7 \\
9 \\
11 \\
18\end{array}$ \\
\hline
\end{tabular}

CSFQ-14: Changes in Sexual Functioning Questionnaire short-form DSM-IV: Diagnosis and Statistical Manual for Mental Disorder, $4^{\text {th }}$

PFSF: Profile of Female Sexual Function

IIEF: International Index of Erectile Function

BMSFI: Brief Male Sexual Function Inventory

Table 2. Characteristics of American studies of sexual dysfunction

\begin{tabular}{|c|c|c|c|c|c|}
\hline & Problem or question & $\begin{array}{l}\text { Sample } \\
\text { size }\end{array}$ & Scale & $\begin{array}{l}\text { Cohort } \\
\text { age } \\
\text { (year) }\end{array}$ & $\begin{array}{c}\text { Prevalence } \\
(\%)\end{array}$ \\
\hline \multicolumn{6}{|l|}{ Female } \\
\hline Chen et al, 2003 & Dyspareunia & 112 & $\begin{array}{c}\text { Ad hoc } \\
\text { questionnaire }\end{array}$ & $40-55$ & 44 \\
\hline Lau et al, 2005 & $\begin{array}{c}\text { Pain disorder } \\
\text { Lubrication problems } \\
\text { No orgasm } \\
\text { No pleasure }\end{array}$ & 3257 & DSM-IV & $18-59$ & $\begin{array}{l}11.4 \\
23.7 \\
13.1 \\
16.2\end{array}$ \\
\hline $\begin{array}{l}\text { Laumann et al, } \\
2005\end{array}$ & $\begin{array}{l}\text { Lack of sexual interest } \\
\text { Inability to reach orgasm } \\
\text { Orgasm too quickly } \\
\text { Pain during sex } \\
\text { Lubrication difficulties }\end{array}$ & 2106 & \begin{tabular}{|c|} 
Ad hoc \\
questionnaire
\end{tabular} & $40-80$ & $\begin{array}{c}39 \\
36.7 \\
21.9 \\
30.4 \\
36\end{array}$ \\
\hline Safarinejad 2006 & $\begin{array}{c}\text { Overall female sexual dysfunction } \\
\text { Orgasmic disorder } \\
\text { Orgasmic disorder } \\
\text { Arousal disorders } \\
\text { Pain disorders }\end{array}$ & 2626 & FSFI & $20-60$ & $\begin{array}{c}31.5 \\
37 \\
35 \\
30 \\
26.7\end{array}$ \\
\hline
\end{tabular}




\begin{tabular}{|c|c|c|c|c|c|}
\hline $\begin{array}{c}\text { Najafabady et al, } \\
2011\end{array}$ & Anorgasmia & 1200 & FSFI & $20-40$ & 26.3 \\
\hline Hisasue et al, 2005 & $\begin{array}{l}\text { Orgasmic disorder } \\
\text { Sexual desire disorder } \\
\text { Arousal disorder } \\
\text { Lubrication disorder } \\
\text { Orgasmic disorder } \\
\text { Sexual desire disorder } \\
\text { Arousal disorder } \\
\text { Lubrication disorder } \\
\end{array}$ & 5042 & $\begin{array}{c}\text { Ad hoc } \\
\text { questionnaire }\end{array}$ & $\begin{array}{l}17-40 \\
17-40 \\
17-40 \\
17-40 \\
40-70 \\
40-70 \\
40-70 \\
40-70\end{array}$ & $\begin{array}{l}15.2 \\
32.2 \\
27.7 \\
57.9 \\
29.7 \\
57.9 \\
12.5 \\
51.2\end{array}$ \\
\hline Singh et al, 2009 & $\begin{array}{c}\text { Difficulties with desire } \\
\text { Arousal disorder } \\
\text { Lubrication problem } \\
\text { Orgasmic disorder } \\
\text { Pain disorder }\end{array}$ & 149 & FSFI & $18+$ & $\begin{array}{l}77.2 \\
91.3 \\
96.6 \\
86.6 \\
64.4\end{array}$ \\
\hline Moreira et al, 2001 & $\begin{array}{l}\text { Lack of sexual pleasure } \\
\text { Inability to reach orgasm } \\
\text { Lubrication difficulties } \\
\text { Pain during sex } \\
\text { Lack of sexual interest }\end{array}$ & 426 & $\begin{array}{c}\text { Ad hoc } \\
\text { questionnaire }\end{array}$ & $40-80$ & $\begin{array}{c}37 \\
31 \\
29.4 \\
28.1 \\
26.9\end{array}$ \\
\hline Sidi et al, 2007 & $\begin{array}{c}\text { Lack of orgasms } \\
\text { Low sexual arousal } \\
\text { Lack of lubrication } \\
\text { Sexual dissatisfaction } \\
\text { Sexual pain }\end{array}$ & 230 & FSFI & $18-70$ & $\begin{array}{l}59.1 \\
60.9 \\
50.4 \\
52.2 \\
67.8\end{array}$ \\
\hline Sobhgol et al, 2007 & Dyspareunia & 319 & $\begin{array}{c}\text { Ad hoc } \\
\text { questionnaire }\end{array}$ & $15-49$ & 54.5 \\
\hline $\begin{array}{c}\text { Goshtasebi et al, } \\
2009\end{array}$ & $\begin{array}{l}\text { Overall sexual dysfunction } \\
\text { Desire difficulty } \\
\text { Arousal difficulty } \\
\text { Lubrication difficulty } \\
\text { Orgasmic difficulty } \\
\text { Pain difficulty } \\
\text { Satisfaction difficulty } \\
\end{array}$ & 1456 & $\begin{array}{c}\text { Ad hoc } \\
\text { questionnaire }\end{array}$ & $15+$ & $\begin{array}{c}52 \\
19.3 \\
18.6 \\
11.9 \\
21.3 \\
18.2 \\
19.4\end{array}$ \\
\hline \multicolumn{6}{|l|}{ Male } \\
\hline Lau et al, 2005 & $\begin{array}{c}\text { Pain disorder } \\
\text { Erectile problems } \\
\text { Premature orgasm } \\
\text { No orgasm } \\
\end{array}$ & 1516 & DSM-IV & $18-59$ & $\begin{array}{c}3.4 \\
9.6 \\
29.7 \\
7.2\end{array}$ \\
\hline $\begin{array}{l}\text { Laumann et al, } \\
2005\end{array}$ & $\begin{array}{c}\text { Lack of sexual interest } \\
\text { Inability to reach orgasm } \\
\text { Early ejaculation } \\
\text { Pain during sex } \\
\text { Erectile difficulties }\end{array}$ & 2701 & $\begin{array}{c}\text { Ad hoc } \\
\text { questionnaire }\end{array}$ & $40-80$ & $\begin{array}{c}23.8 \\
19.1 \\
29.8 \\
8.9 \\
27.6\end{array}$ \\
\hline Nicolosi et al 2003 & Erectile dysfunction & 600 & $\begin{array}{c}\text { Ad hoc } \\
\text { questionnaire } \\
\end{array}$ & $40-70$ & 34 \\
\hline
\end{tabular}




\begin{tabular}{|c|c|c|c|c|c|}
\hline Junior et al, 2005 & $\begin{array}{c}\text { Early ejaculation } \\
\text { Inability to reach orgasm } \\
\text { Erectile difficulties } \\
\text { Lack of sexual interest }\end{array}$ & 471 & \begin{tabular}{|c|} 
Ad hoc \\
questionnaire
\end{tabular} & $40-80$ & $\begin{array}{c}30.3 \\
14 \\
13.1 \\
11.2 \\
\end{array}$ \\
\hline Moreira et al, 2001 & $\begin{array}{c}\text { Early ejaculation } \\
\text { Erectile difficulties } \\
\text { Lack of sexual interest } \\
\text { Inability to reach orgasm } \\
\text { Sex not pleasurable } \\
\end{array}$ & 546 & $\begin{array}{c}\text { Ad hoc } \\
\text { questionnaire }\end{array}$ & $40-80$ & $\begin{array}{l}32.7 \\
31.9 \\
28.3 \\
19.3 \\
18.1 \\
\end{array}$ \\
\hline $\begin{array}{c}\text { Kongkanand et al, } \\
2000 \\
\end{array}$ & Erectile difficulties & 1259 & $\begin{array}{c}\text { Ad hoc } \\
\text { questionnaire }\end{array}$ & $40-70$ & 37.5 \\
\hline Marumu et al, 2001 & Erectile difficulties & 1517 & IIEF & $\begin{array}{l}23-29 \\
30-39 \\
40-49 \\
50-59 \\
60-69 \\
70-79 \\
\end{array}$ & $\begin{array}{c}19.2 \\
2.3 \\
9.5 \\
15.7 \\
34.4 \\
53.4\end{array}$ \\
\hline
\end{tabular}

DSM-IV: Diagnosis and Statistical Manual for Mental Disorder, $4^{\text {th }}$ FSFI: Female Sexual Function Index

IIEF: International Index of Erectile Function

Table 3. Characteristics of Asian studies of sexual dysfunction

\begin{tabular}{|c|c|c|c|c|c|}
\hline & Problem or question & $\begin{array}{l}\text { Sample } \\
\text { size }\end{array}$ & Scale & $\begin{array}{l}\text { Cohort } \\
\text { age } \\
\text { (year) }\end{array}$ & $\begin{array}{c}\text { Prevalence } \\
\quad(\%)\end{array}$ \\
\hline \multicolumn{6}{|l|}{ Female } \\
\hline $\begin{array}{c}\text { Dennerstein et al, } \\
2006\end{array}$ & $\begin{array}{l}\text { Hypoactive sexual desire } \\
\text { disorder }\end{array}$ & 2467 & PFSF & $50-70$ & 12 \\
\hline Laumann et al, 2005 & $\begin{array}{c}\text { Lack of sexual interest } \\
\text { Inability to reach orgasm } \\
\text { Orgasm too quickly } \\
\text { Pain during sex } \\
\text { Lubrication difficulties }\end{array}$ & 3494 & $\begin{array}{c}\text { Ad hoc } \\
\text { questionnaire }\end{array}$ & $40-80$ & $\begin{array}{c}27.6 \\
20.9 \\
9.6 \\
10.4 \\
17.2\end{array}$ \\
\hline Weiss et al, 2009 & Arousal Disorder & 1000 & $\begin{array}{c}\text { Ad hoc } \\
\text { questionnaire }\end{array}$ & $15-88$ & 10.3 \\
\hline $\begin{array}{c}\text { Ponholzer et al, } \\
2004\end{array}$ & $\begin{array}{l}\text { desire disorders } \\
\text { arousal disorders } \\
\text { orgasmic problems } \\
\text { Pain disorders }\end{array}$ & 703 & $\begin{array}{c}\text { Ad hoc } \\
\text { questionnaire }\end{array}$ & $20-80$ & $\begin{array}{c}22 \\
35 \\
39 \\
12.8\end{array}$ \\
\hline Hayes et al, 2008 & $\begin{array}{c}\text { hypoactive sexual desire } \\
\text { disorder } \\
\text { sexual arousal disorder } \\
\text { (lubrication) } \\
\text { orgasmic disorder } \\
\text { dyspareunia }\end{array}$ & 356 & SFQ & $20-70$ & $\begin{array}{l}16 \\
7 \\
8 \\
1\end{array}$ \\
\hline Mercer et al, 2003 & Lack of interest in sex & 11161 & ICD-10 & $16-44$ & 40.6 \\
\hline
\end{tabular}




\begin{tabular}{|c|c|c|c|c|c|}
\hline & $\begin{array}{c}\text { Unable to experience } \\
\text { orgasm } \\
\text { Premature orgasm } \\
\text { Painful intercourse } \\
\text { Trouble lubricating }\end{array}$ & & & & $\begin{array}{c}14.4 \\
1.3 \\
11.8 \\
9.2\end{array}$ \\
\hline Štulhofer et al, 2005 & $\begin{array}{c}\text { experienced sexual } \\
\text { problems } \\
\text { inhibited desire } \\
\text { Inhibited arousal } \\
\text { inhibited orgasm } \\
\text { Dyspareunia } \\
\end{array}$ & 547 & $\begin{array}{c}\text { Ad hoc } \\
\text { questionnaire }\end{array}$ & $20-60$ & $\begin{array}{c}33.8 \\
11.2 \\
12.1 \\
18.4 \\
6.4\end{array}$ \\
\hline Dunn et al, 1998 & $\begin{array}{l}\text { Orgasmic Dysfunction } \\
\text { Dyspareunia } \\
\text { Vaginal Dryness } \\
\text { problem with arousal } \\
\text { inhibited enjoyment }\end{array}$ & 979 & $\begin{array}{c}\text { Ad hoc } \\
\text { questionnaire }\end{array}$ & $18-75$ & $\begin{array}{l}27 \\
18 \\
18 \\
17 \\
18\end{array}$ \\
\hline Oksuz et al, 2006 & $\begin{array}{l}\text { overall sexual dysfunction } \\
\text { desire problem } \\
\text { arousal problem } \\
\text { lubrication problem } \\
\text { orgasm problem } \\
\text { pain problem }\end{array}$ & 518 & FSFI & $18-55$ & $\begin{array}{l}48.3 \\
48.3 \\
35.9 \\
40.9 \\
42.7 \\
42.9\end{array}$ \\
\hline $\begin{array}{l}\text { Danielsson et al, } \\
2003\end{array}$ & Dyspareunia & 3017 & $\begin{array}{c}\text { Ad hoc } \\
\text { questionnaire }\end{array}$ & $\begin{array}{l}20-60 \\
20-29 \\
30-39 \\
40-49 \\
50-60 \\
\end{array}$ & $\begin{array}{l}9.3 \\
13 \\
10 \\
8.6 \\
6.5\end{array}$ \\
\hline TrÆen et al, 2010 & $\begin{array}{c}\text { Reduced sexual desire } \\
\text { Problem achieving orgasm } \\
\text { Genital pain }\end{array}$ & 744 & $\begin{array}{c}\text { Ad hoc } \\
\text { questionnaire }\end{array}$ & $18-67$ & $\begin{array}{c}37 \\
26 \\
9\end{array}$ \\
\hline \multicolumn{6}{|l|}{ Male } \\
\hline Braun et al, 2000 & erectile dysfunction & 4489 & $\begin{array}{c}\text { Ad hoc } \\
\text { questionnaire }\end{array}$ & $\begin{array}{l}30-80 \\
30-39 \\
40-49 \\
50-59 \\
60-69 \\
70-80 \\
\end{array}$ & $\begin{array}{c}19.2 \\
2.3 \\
9.5 \\
15.7 \\
34.4 \\
53.4 \\
\end{array}$ \\
\hline Laumann et al, 2005 & $\begin{array}{l}\text { Lack of sexual interest } \\
\text { Inability to reach orgasm } \\
\text { Early ejaculation } \\
\text { Pain during sex } \\
\text { Erectile difficulties }\end{array}$ & 4311 & $\begin{array}{c}\text { Ad hoc } \\
\text { questionnaire }\end{array}$ & $40-80$ & $\begin{array}{c}12.7 \\
10.6 \\
21.1 \\
3.6 \\
13.1\end{array}$ \\
\hline Nicolosi et al 2003 & erectile dysfunction & 600 & $\begin{array}{c}\text { Ad hoc } \\
\text { questionnaire }\end{array}$ & $40-70$ & 17 \\
\hline Mercer et al, 2003 & $\begin{array}{c}\text { Lack of interest in sex } \\
\text { Unable to experience } \\
\text { orgasm } \\
\text { Premature orgasm }\end{array}$ & 11161 & ICD-10 & $16-44$ & $\begin{array}{c}17.1 \\
5.3 \\
11.7 \\
17 \\
\end{array}$ \\
\hline
\end{tabular}




\begin{tabular}{|c|c|c|c|c|c|}
\hline & $\begin{array}{l}\text { Painful intercourse } \\
\text { Unable to achieve or } \\
\text { maintain erection }\end{array}$ & & & & 5.8 \\
\hline Akkus et al, 2002 & erectile dysfunction & 1982 & $\begin{array}{c}\text { Ad hoc } \\
\text { questionnaire }\end{array}$ & $40+$ & 69.2 \\
\hline Dunn et al, 1998 & $\begin{array}{l}\text { Difficulty getting erection } \\
\text { Difficulty maintaining } \\
\text { erection } \\
\text { erectile dysfunction } \\
\text { Premature ejaculation } \\
\text { inhibited enjoyment }\end{array}$ & 789 & $\begin{array}{c}\text { Ad hoc } \\
\text { questionnaire }\end{array}$ & $18-75$ & $\begin{array}{c}21 \\
24 \\
26 \\
14 \\
9\end{array}$ \\
\hline Giuliano et al, 2002 & erectile dysfunction & 1004 & IIEF-5 & $\begin{array}{c}40+ \\
40-49 \\
50-59 \\
60-69 \\
\geq 70\end{array}$ & $\begin{array}{c}31.6 \\
32.2 \\
27 \\
19.7 \\
21\end{array}$ \\
\hline Parazzini et al, 2000 & Erectile Dysfunction & 2010 & $\begin{array}{c}\text { Ad hoc } \\
\text { questionnaire }\end{array}$ & $\begin{array}{c}18+ \\
18-29 \\
30-39 \\
40-49 \\
50-59 \\
60-70 \\
>70\end{array}$ & $\begin{array}{c}12.8 \\
2.8 \\
1.9 \\
4.8 \\
15.7 \\
26.8 \\
48.3\end{array}$ \\
\hline $\begin{array}{c}\text { Blanker, M et al, } \\
2001\end{array}$ & $\begin{array}{l}\text { Erectile dysfunction } \\
\text { Ejaculatory dysfunction }\end{array}$ & 1688 & $\begin{array}{l}\text { ICS male sex } \\
\text { questionnaire }\end{array}$ & $\begin{array}{l}50-54 \\
55-59 \\
60-64 \\
65-69 \\
70-78 \\
50-54 \\
55-59 \\
60-64 \\
65-69 \\
70-78\end{array}$ & $\begin{array}{c}3 \\
5 \\
11 \\
19 \\
26 \\
3 \\
5 \\
11 \\
21 \\
35\end{array}$ \\
\hline TrÆen et al, 2010 & $\begin{array}{c}\text { Reduced sexual desire } \\
\text { Problem achieving orgasm } \\
\text { Genital pain }\end{array}$ & 873 & Ad hoc questionnaire & $18-67$ & $\begin{array}{c}13 \\
6 \\
2\end{array}$ \\
\hline $\begin{array}{c}\text { Martin-Morales et } \\
\text { al, } 2001\end{array}$ & Erectile dysfunction & 2476 & $\begin{array}{c}\text { IIEF } \\
\text { Simple question }\end{array}$ & $25-70$ & $\begin{array}{l}18.9 \\
12.1\end{array}$ \\
\hline
\end{tabular}

PFSF: Profile of Female Sexual Function

SFQ: Sexual Function Questionnaire

ICD-10: International Classification of Diagnosis-10

FSFI : Female Sexual Function Index

IIEF-5: International Index of Erectile Function-5

IIEF: International Index of Erectile Function

Table 4. Characteristics of European studies of sexual dysfunction 


\section{Conclusions}

Existing epidemiologic data on sexual dysfunction support high prevalence of these problems worldwide. However, the data are limited and the prevalence data on male sexual dysfunction, except for ED, are too limited. Widely accepted definitions of disorders and scales are primary prerequisites to make prevalence comparisons possible and describe the severity of the problem.

\section{Acknowledgement}

Authors would like to thank Ms Zahra Sehat for searching and organizing papers.

\section{References}

Abdo, C. Oliveira, W. Moreira, E. Fittipaldi, J. 2004. Prevalence of sexual dysfunctions and correlated conditions in a sample of Brazilian women-results of the Brazilian study on sexual behavior (BSSB). International Journal of Impotence Research, 2004, 160-166.

Addis, I. Van Den Eeden, S. Wassel-Fyr, C. Vittinghoff, E. Brown, J. Thom, D. 2006. Sexual Activity and Function in Middle-Aged and Older Women. Obstet Gynecol. , 107, 4, 755-764.

Akkus, E. Kadioglo, A.Esen, A. Doran, A. Ergen, A. ans et. al. 2002. prevalence and correlate of erectile dysfunction in Turkey: a population based- study. Eropean Urology, 41,268-304.

Althof, S. 2006.Prevalence, Characteristics and Implications of Premature Ejaculation/Rapid Ejaculation. Journal of Urology, 175, 842-848.

Amidu, N. Owired, W. Gyasi-Sa rpong, C. Wood, E. Quaye, L. 2011. Sexual dysfunction among married couples living in Kumasi metropolis, Ghana. BMC Urology, 11, 3, 3-7.

Ansong, K. Lewis, C. Jenkins, P. Bell, J. 2000. Epidemiology of Erectile Dysfunction: A Community-based Study in Rural New York State. Ann Epidemiol ,10,293-296.

Bancroft, J. Loftus, J., Scott Long, J. 2003. Distress about Sex: A National Survey of Women in Heterosexual Relationships. Archives of Sexual Behavior, 32, 3, 193-208.

Blanker, M. Bosch, J. Grooeneveld, F. Bohnen, A. Thomas, A. Hop, W. 2001.Erectile and ejaculatory dysfunction in a community-based sample of men 50-78 years old: prevalence, concern, and relation to sexual activity. UROLOGY, 57,763-768.

Braun, M. Wassmer, G. Klotz, T. Reifenrath, B. Mathers, M. Engelmann, U. Epidemiology of erectile dysfunction: results of the 'Cologne Male Survey'. 2000. International Journal of Impotence Research,12, 305-311.

Chen , O. 2003. Taneepanichskul, S. Prevalence of Dyspareunia in Healthy Thai Perimenopausal Women. Thai Journal of Obstetrics and Gynaecology, 15, 113-121.

Danielsson,I. berg, I. s Stenlund, H. Wikman, M. 2003. Prevalence and incidence of prolonged and severe dyspareunia in women: results from a population study. Scand J Public Health, 31, 113-118.

Dennerstein, L.Koochaki, P. Barton, I. Graziottin, A. 2006. Hypoactive sexual desire disorder in menopausal women: a survey of western European women. The Journal of Sexual Medicine, 3, 212-222. 
Derby, C. Araujo, A Johannes, C. Feldman, H. McKinlay, J. 2000. Measurement of erectile dysfunction in population-based studies: the use of a single question selfassessment in the Massachusetts Male Aging Study. International Journal of Impotence Research,2000, 12, 197-204.

Dunn, k. Croft, P. Hackett, G. 1998.Sexual problem: a study of the prevalence and need for health care in the general population. Family Practice, 25,6, 519-524.

Elnashar, A. Ibrahim, M. EL-Deso ky, M. Ali, O. 2007. Mohamed Hassan, M. Female sexual dysf unction in Lower Egypt. BJOG, 114, 201 -206.

Giuliani, F. Cheveret- Measson, M. Tsatsaries, A. Reitz, C. Murino, M. thonneau, P. 2002. Prevalnce of erectile dysfunction in France: Result of an Epidemiologic survey of a reproductive sample of 1004 men. Eropean Urology, 42, 382-389.

Goshtasebi, A.Vahdaninia, M. Rahimi Foroshani, A. 2009. Prevalence and Potential Risk Factors of Female Sexual Difficulties: An Urban Iranian Population-Based Study. J Sex Med , 6, 2988-2996.

Hartmann U, Heiser K, Rüffer-Hesse C, Kloth G. Female sexual desire disorders: subtypes, classification, personality factors and new directions for treatment. 2002 World Journal of Urology; 20: 79-88.

Hassanin, I. Helmy, Y. Fathalla, M. Shahin, A. 2010. Prevalence and characteristics of female sexual dysfunction in a sample of women from Upper Egypt. International Journal of Gynecolo gy and Obstetrics 108, 219- 223.

Hayes RD, Dennerstein L, Bennett CM, Sidat M, Gurrin LC, and Fairley CK. Risk factors for female sexual dysfunction in the general population: Exploring factors associated with low sexual function and sexual distress. J Sex Med 2008;5:1681-1693.

Hisasue, S. kumamoto, Y. Sato, Y. Masumoori, N. Horit, H. and et al. 2005. Prevalence of female sexual dysfunction symptoms and its relationship to quality of life: A Japanese female cohort study. UROLOGY, 65,143-148.

Jonannes, c. Araujo, A. Feldman, H. Derby, C. 2000. Kleinnman, K. Mckinlay, J. Incidence of Erectile Dysfunction in Men 40 To 69 Years Old: Longitudinal Results from the Massachusetts Male Aging Study. The Journal of Urology, 163, 460 - 463.

Junior, E. Glasser, D. Santos, D. Gingell, C. 2005.Prevalence of sexual problems and related help-seeking behaviors among mature adults in Brazil: data from the Global Study of Sexual Attitudes and Behaviors. Sao Paulo Med J.123, 5, 234-41.

Kadri, K. Alami, M. Tahiri, M. 2002, Sexual dysfunction in women: population based epidemiological study. Arch Womens Ment Health , 5,59-63.

Kinsey AC, Pomeroy WB, Martin CE and Gebhard PH. Sexual Behavior in the Human Female. W. B. Saunders \& Co. Philadelphia 1953.

Kongkanand, A. 2000. Prevalence of erectile dysfunction in Thailand. International Journal of Andrology, 23, 2, 77-80.

Lau, J. Kim JH. Tsui, H-Y. 2005. Prevalence of male and female sexual problems, perceptions related to sex and association with quality of life in a Chinese population: a population-based study. International Journal of Impotence Research, 17, 494-505.

Laumann, E. Das, A. Waite, L. 2008. Sexual Dysfunction among Older Adults: Prevalence and Risk Factors from a Nationally Representative U.S. Probability Sample of Men and Women 57-85 Years of Age. J SexMed ,5, 10, 2300-2311.

Laumann, E. Paik, A. Rosen, R.1999. Sexual Dysfunction in the United States Prevalence and Predictors. JAMA, 2, 81:537-544. 
Laumann, EO. Nicolosi, A. Glasser, DB. Paik, A. Gingell, C. Moreira, E. Wang, T. 2005. Sexual problems among women and men aged 40-80 y: prevalence and correlates identified in the Global Study of Sexual Attitudes and Behaviors. International Journal of Impotence Research,17, 39-57.

Lindau, s. Schumm, P. Laumann, O. Levinson, W. O'Muircheartaigh, C. Waite, L. 2007. A Study of Sexuality and Health among Older Adults in the United States.. N Engl J Med, 357, 22-34.

Marumu, K. Nakashima, J. Murai, M.2001. Age related prevalence of erectile dysfunction in Japan. Assessment by the international index of erectile dysfunction. International Journal of Urology, 8, 53-59.

Mercer, C. Fenton, K. Johnson, A. Wellings, K. Macdowall, W. McManus, S. Nanchahal, K. Erens, B. 2003.Sexual function problems and help seeking behavior in Britain: national probability sample survey. BMJ. 327, 23, 426-427.

Martin-Morales A, Sanchez-Cruz JJ, Saenz de Tejada I, Rodriguez-Vela L, Jimenez-Cruz JF, Burgos-Rodriguez R. 2001. Prevalence and independent risk factors for erectile dysfunction in Spain: results of the Epidemiologia de la Disfuncion Erectil Masculina Study. Journal of urology,166,569-74.

Moreira, E. Adbo, C. Torres, E. Lobo, C. Fittipaldi, N.2001. prevalence and correlates of erectile dysfunction: result of the Brazilian study of sexual behavior. UROLOGY, $58,583-588$.

Najafabady MT, Salmani Z, Abedi P. 2011. Prevalence and related factors for anorgasmia among reproductive aged women in Hesarak, Iran. Clinics (Sao Paulo); 66, 83-6.

Nicolosi, A. Morejra, E. Shirai, M. Tambi, M. Glasser, D. 2003.Epidemiology of erectile dysfunction in four countries: cross-national study of the prevalence and correlates of erectile dysfunction. J of Urology, 61,1, 201-206.

Oksuz, E. Malhan, S. 2006. Prevalence and Risk Factors for Female Sexual Dysfunction in Turkish Women. THE JOURNAL OF UROLOGY, 175, 654-658.

Parazzini F, Menchini Fabris F, Bortolotti A, Calabrò A, Chatenoud L, Colli E, Landoni M, Lavezzari M, Turchi P, Sessa A, Mirone V. 2000. Frequency and determinants of erectile dysfunction in Italy. Eur Urol;37:43-9.

Prins, J. Blanker, MH. Bohnen, AM. Thomas, S. Bosch, J. 2002. Prevalence of erectile dysfunction: a systematic review of population-based studies. International Journal of Impotence Research, 14, 422-432.

Ponholzera, A.Roehlicha, M. Racza, U. Temmla, C. Madersbacher, S. 2005. Female Sexual Dysfunction in a HealthyAustrian Cohort: Prevalence and Risk Factors. European Urology, 47, 366-375.

Safarinejad, MR. 2006. Female sexual dysfunction in a population-based study in Iran: prevalence and associated risk factors. International Journal of Impotence Research, 18, 382-395

Saigal, C. Wessells, H. Pace, J. Schonlau, M.Wilt, T. 2006. Predictors and Prevalence of Erectile Dysfunction in a Racially Diverse Population. Arch Intern Med,166,207-212.

Seyam, R. Albakry, A.Ghobish, A. Arif, H. Dandash, K. Rashwan, H. 2003.Prevalence of erectile dysfunction and its correlates in Egypt: a community-based study. International Journal of Impotence Research, 15, 237-245.

Shifren, J. Monz, B. Russo, P. Segreti, A. Johannes, C. 2008. Sexual Problems and Distress in United States Women: Prevalence and Correlates. Obstet Gynecol, 112, 970-8. 
Sidi, H. Puteh, S.Abdullah, N. Midin, M. 2007. The Prevalence of Sexual Dysfunction and Potential Risk Factors That May Impair Sexual Function in Malaysian Women. J Sex Med, 4, 311-321.

Simons, J. Carey, M.2001. Prevalence of Sexual Dysfunctions: Results from a Decade of Research. Arch Sex Behav, 30, 2, 177-219.

Singh, j. Tharyan, P. Kekre, N. Singh, G. Gopalakrishnan, G. 2009. Prevalence and risk factors for female sexual dysfunction in women attending a medical clinic in south India. Journal of Post Gratuate Medicine, 55, 2, 113-120.

Sobhgol, S.Mohammad Alizadeli Charndabee, S. 2007. Rate and related factors of dyspareunia in reproductive age women: a cross-sectional study. International Journal of Impotence Research,19, 88-94.

Štulhofer,A. Greguroviæ, M. Pikiæ, A. Galiæ, A. 2005. Sexual Problems of Urban Women in Croatia: Prevalence and Correlates in a Community Sample. Croat Med J, 46, $1,45-51$.

Tiefer, L. 2001. A new view of women's sexual problem: why new? why now?. J Sex Research, 38, 89-96.

Træen, B. Stigun, H. 2010. Sexual problems in 18-67-year-old Norwegians. Scandinavian Journal of Public Health, 38, 445-456.

Tsai, T. Yeh, C. Hwang, T. 2011. Female Sexual Dysfunction: Physiology, Epidemiology, Classification, Evaluation and Treatment. Urol Sci, 22, 1, 7-13.

Weiss, P. Brody, S. 2009. Female Sexual Arousal Disorder with and without a Distress Criterion: Prevalence and Correlates in a Representative Czech Sample. J Sex Med, 6, 3385-3394.

West, S. D'Aloisio, A. Agans, R. Kalsbeek, W. Borisov, N. Thorp, J. 2008. Prevalence of Low Sexual Desire and Hypoactive Sexual Desire Disorder in a Nationally Representative Sample of US Women. Arch Intern Med, 168, 13,1441-1449. 


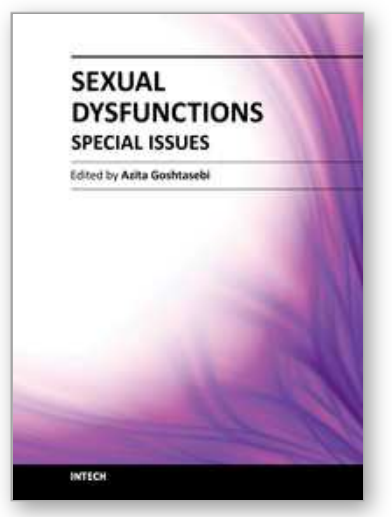

\author{
Sexual Dysfunctions - Special Issues \\ Edited by Dr. Azita Goshtasebi
}

ISBN 978-953-307-859-5

Hard cover, 128 pages

Publisher InTech

Published online 22, December, 2011

Published in print edition December, 2011

Sexual dysfunctions have recently recognized as one of the major public health problems. This book enhances our scientific understanding of sexual function and dysfunction from different perspectives. It presents evidence-based interventions for sexual dysfunctions in difficult medical situations such as cancer, and gives a valuable overview of recent experimental researches on the topic. Published in collaboration with InTech Open Access Publisher, this imperative work will be a practical resource for health care providers and researchers who are involved in the study of sexual health.

\title{
How to reference
}

In order to correctly reference this scholarly work, feel free to copy and paste the following:

Azita Goshtasebi, Samira Behboudi Gandevani, and Abbas Rahimi Foroushani (2011). Prevalence of Sexual Dysfunctions: A Systemic Approach, Sexual Dysfunctions - Special Issues, Dr. Azita Goshtasebi (Ed.), ISBN: 978-953-307-859-5, InTech, Available from: http://www.intechopen.com/books/sexual-dysfunctions-specialissues/prevalence-of-sexual-dysfunctions-a-systemic-approach

\section{INTECH}

open science | open minds

\section{InTech Europe}

University Campus STeP Ri

Slavka Krautzeka 83/A

51000 Rijeka, Croatia

Phone: +385 (51) 770447

Fax: +385 (51) 686166

www.intechopen.com

\section{InTech China}

Unit 405, Office Block, Hotel Equatorial Shanghai

No.65, Yan An Road (West), Shanghai, 200040, China

中国上海市延安西路65号上海国际贵都大饭店办公楼 405 单元

Phone: +86-21-62489820

Fax: +86-21-62489821 
(C) 2011 The Author(s). Licensee IntechOpen. This is an open access article distributed under the terms of the Creative Commons Attribution 3.0 License, which permits unrestricted use, distribution, and reproduction in any medium, provided the original work is properly cited. 